\title{
Use of Unlocked Intramedullary Nailing in Winquist Type I and II Femoral Isthmus Fracture
}

\author{
HT Ling, MBBS (UM), WM Ng, MS Ortho (UM), MK Kwan, MS Ortho (UM), \\ LK Fathi Aizuddeen, MBBS (UM), PCM Tay, MBBS (UM) \\ Department of Orthopaedic Surgery, University Malaya Medical Center, Kuala Lumpur, Malaysia
}

\begin{abstract}
Interlocked intramedullary nailing is accepted as the gold standard for femoral shaft fractures. However for Winquist type I and II femoral fractures at the isthmus region, unlocked intramedullary nailing (Küntscher nailing) is still a good option. We performed a retrospective study on 86 patients with a total of 88 femoral shaft fractures around the isthmus that presented at our institution between 1 January 1988 and 31 August 2003. All patients (84.1\% Winquist type I and $15.9 \%$ Winquist Type II fractures) were treated with unlocked intramedullary nail. The average time to union was 16 weeks with $97.7 \%$ rate of union. There were two cases (2.3\%) of infection and non-union each. Overall results were comparable to standard interlocking intramedullary nailing. We conclude that unlocked intramedullary nailing is a good treatment option for Winquist Type I and II femoral fracture around the isthmus with its good union rate and minimal complications.
\end{abstract}

Unlocked intramedullary nailing, Küntscher nailing, Femoral fracture

\section{INTRODUCTION} nailing for femoral fractures in the 1940's. The inherent rotational instability of unlocked intramedullary nailing was solved with the introduction of the locked intramedullary nail in the1970's. In 1984, Winquist RA et al ${ }^{1}$ in their study of a series of intramedullary nailing of femoral fractures concluded that unlocked intramedullary nailing should only be used for the middle one third femoral shaft fracture with Winquist type I or type II, similar to the results of Hooper GJ and Lyon $\mathrm{DW}^{2}$ published in 1988.

In our centre, patients who present with either Winquist type I or II femoral diaphyseal fracture over the isthmus region will be treated with unlocked intramedullary nailing (Küntscher nail). Reasons for use of the unlocked intramedullary nail include: the cost of the implant is $20 \%$ of

\section{Key Words:}

Gerhard Küntscher first introduced unlocked intramedullary

the cost locked intramedullary nail, the procedure can be done without use of the image intensifier in relatively short operative time and the clinical outcome is comparable to that of locked intramedullary nailing. In this study, we re-assess the results of our cases treated with the unlocked intramedullary nail.

\section{MATERIALS AND METHODS}

Patients treated for femoral shaft fractures between 1st January 1988 and 31st August 2003 were reviewed retrospectively. Medical records and radiographs were reviewed and those with Winquist type I and II femoral fractures treated with unlocked intramedullary nail (Küntscher nail) fixation were included in the study. All patients had at least one-year follow-up (range, 12 months to 60 months).

Most patients were admitted through the emergency/trauma unit, where the injured limb was placed on Böhler Braun frame and stabilized with traction of 5-10 kg using a high tibial pin. If the patient was expected to undergo surgery within a few days, skin traction was used. Surgeries were performed by the specialist or senior registrar. Most patients were allowed to mobilize early in the recover period, except those with bilateral fractures or other limiting concomitant injuries. Partial weight bearing with crutches and full range of motion exercise of the knee was encouraged. Patients were allowed full weight bearing when there was clinical and radiological evidence of fracture union.

Operative treatment
All patients were placed in lateral position for the operative
procedure. A direct lateral approach was used to reach the
fracture site. Periosteum was elevated just enough to allow
reduction of the fracture. The hip was flexed to 90 degree
and the proximal medullary canal was hand reamed in
retrograde direction toward the piriformis fossa. Before the
piriformis fossa was perforated, manual measurement of the
proximal bone length from the fracture site to the piriformis
fossa was performed using the corresponding reamer length.
The length of the distal bone fragment was measured from Corresponding Author: Ng Wuey Min, Department of Orthopaedic Surgery, University Malaya Medical Center, 50603 Kuala Lumpur, Malaysia Email: wueymin_ng@hotmail.com 
the fracture site to maximum depth of the distal medullary canal, which usually corresponds to the level of patella. The medullary canal of both the proximal and the distal segment of the femur were reamed sequentially with manual reamers in increments of $0.5 \mathrm{~mm}$ diameter. Care was taken not to break the fracture ends. The reaming process was performed until the surgeon felt resistance at the isthmus cortical bone.

An unlocked intramedullary nail (Küntscher nail) with the diameter equal to that of the last reamer was used. The hip was kept in 90-degree flexion, and using a mallet, the nail was inserted into the medullary canal of the proximal femoral segment in a retrograde fashion, until it emerged from the piriformis fossa and tented the gluteal skin. The skin was then incised and the nail was allowed to protrude until the distal end of the nail was flush with the fracture site. The fracture was reduced manually and the nail was hammered antegradely until the proximal end of the nail reached the level of the greater trochanter.

For patients with fracture of both femurs, the procedure was conducted similarly on each side. The side fixated first, was covered with compression bandage and the patient turned for nailing of the other side.

\section{RESULTS}

There were 86 patients with a total of 88 femoral diaphyseal fractures treated with the unlocked intramedullary nail (Küntscher nail) during the study period. Most patients (84.9\%) sustained the injury from a motor vehicle accident. There were $78(90.7 \%)$ males and $8(9.3 \%)$ females, with roughly equal numbers of right and left femurs involved. Of the 88 fractures, $75(85.2 \%)$ were of Winquist type I and 13 $(14.8 \%)$ were of Winquist type II. There were 2 open fractures classified as Gustilo type I. The mean age of the patient was 23 years and a majority were aged between 16 and 25 years (Fig. 1).

The average time from injury to surgery was 5.2 days (range 0-17 days). Eleven patients (who had bilateral femoral fractures, multiple long bones fractures or open fracture of other bones) had the intramedullary nailing performed on the day of admission. Two patients with open femoral fractures had emergency surgical debridement of the wound on the day of admission and intramedullary nailing was performed after day 14 and 15 days respectively. Seventy-nine (89.8\%) femurs were fixed with nail length between $36-42 \mathrm{~cm}$ (Fig. 2 ). Seventy-eight $(88.6 \%)$ femurs were fixed with nails of diameter between 9 and $12 \mathrm{~mm}$ (Fig. 3). Intraoperative iatrogenic fracture of bone ends occurred in 3 patients (3.4\%). One of these patients developed nonunion while the other two united uneventfully. There were no systemic complications such as fat embolism noted among study subjects.
Union was achieved in 86 out of 88 fractures (97.7\%) after an average of 111 days ( $\mathrm{SD} \pm 58$ days). Nonunion occurred in 2 patients $(2.3 \%)$. One patient underwent exchanged intramedullary nail 11 months post-trauma and the fracture was united three months later. In the other patient the Winquist type I fracture was converted to type III fracture during the reaming procedure. Two cerclage wires were used to fix the butterfly fragment and the procedure was continued. There was no radiographic evidence of union after 5 months and exchanged intramedullary nailing was then performed. After 3 years, there was still no evidence of union nor sign of infection. Eventually the decision was made to remove the nail and fix the fracture using a locking compression plate with bone grafting. The fracture united 4 months after the latter procedure.

Infection was encountered in 2 patients $(2.3 \%)$. The first patient sustained an open fractured femur. He presented with discharging sinus from the wound 5 months after the insertion of the unlocked intramedullary nail. Surgical debridement of the sinus, removal of implant and reaming of the intramedullary canal was performed. The fracture was found to have united intraoperatively. Histopathology report of bone and tissue removed during surgery suggested osteomyelitis, and scant growth of Pseudomonas aeruginosa was cultured. There was no further sign of infection following six weeks of intravenous antibiotics. The other patient was a 14 year-old boy who had a closed fracture of the right femur. He had fever post operatively for five days. The wound developed haemo-serous discharge and the swab culture grew Acinetobacter. He was discharged with two weeks of oral antibiotics. He presented with a small discharging sinus from the wound four months after the surgery. The fracture had united. He underwent surgical debridement, removal of nail and reaming of intramedullary canal. Tissue send from the operative site grew Staphylococcus aureus, and the patient was then was treated with six weeks of intravenous antibiotics.

A bent Küntscher nail was noted in two patients (2.3\%). Both of them had nails of $10 \mathrm{~mm}$ diameter. Both nails were noted to be bent approximately two months after surgery when they were allowed full weight bearing ambulation. They had bone union at 3 months and 6 months respectively. One nail was bent about six degrees resulting in varus deformity over the fracture site and the other nail was bent about four degrees with no gross femoral deformity noted. Both nails were removed at 15 months and 29 months after surgery. The nails were extracted without disrupting the bone. No nail fracture was encountered. Four patients $(4.5 \%)$ were documented to have one centimetre shortening but none of them need modification of shoe wear. In this study, we did not measure rotational deformity. 


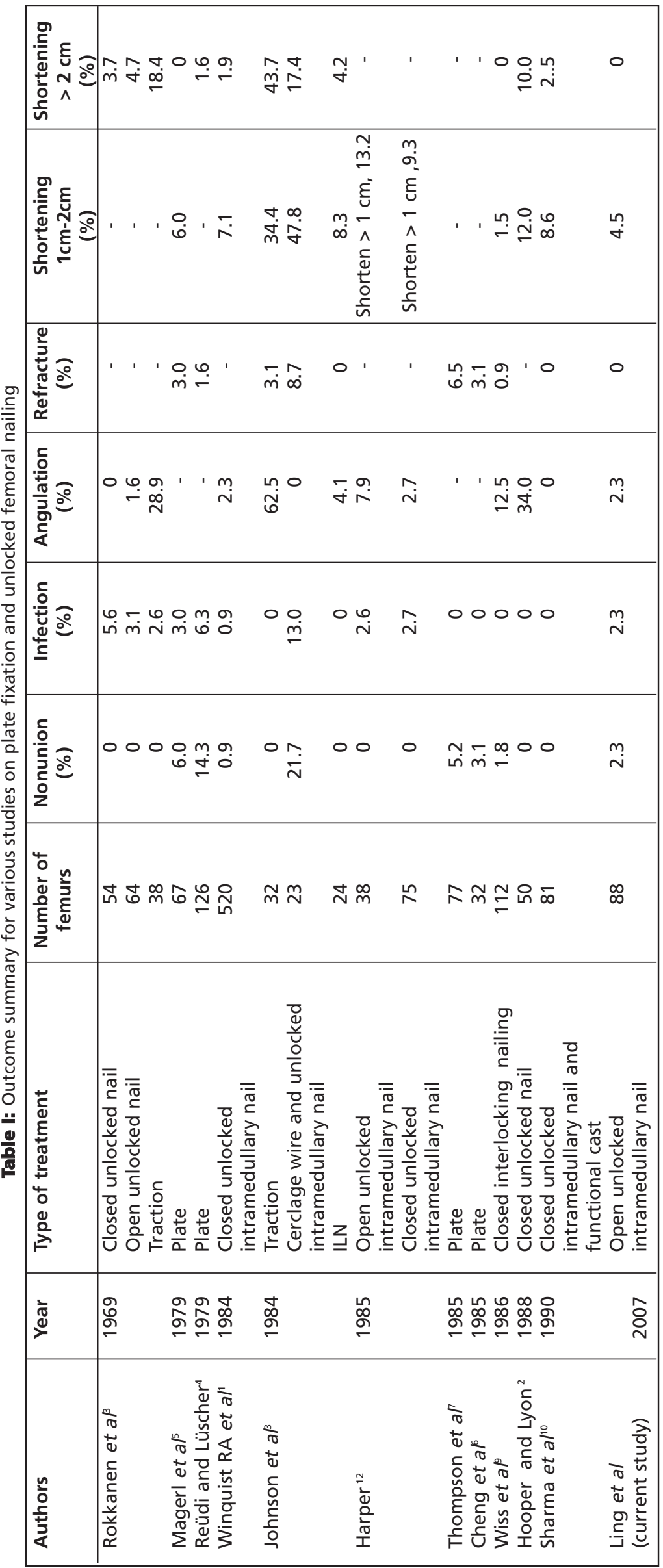




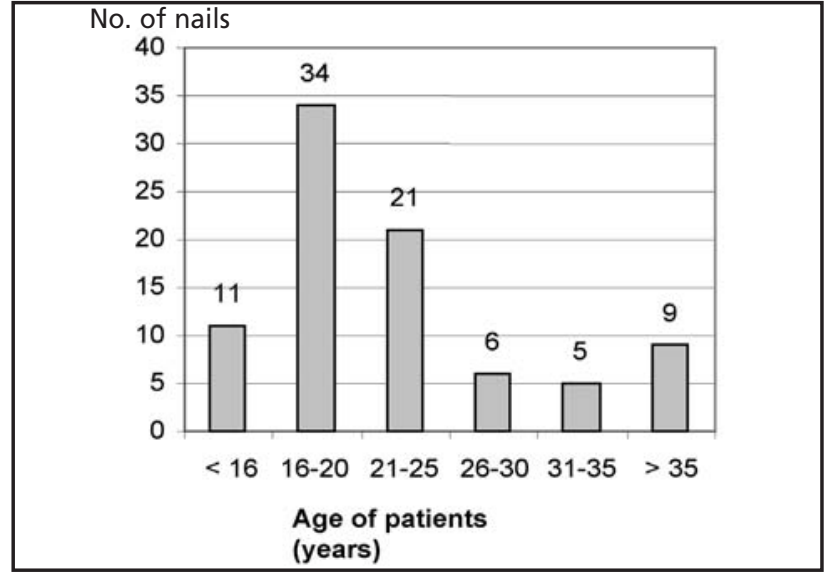

Fig. 1: Age distribution of patients in this study.

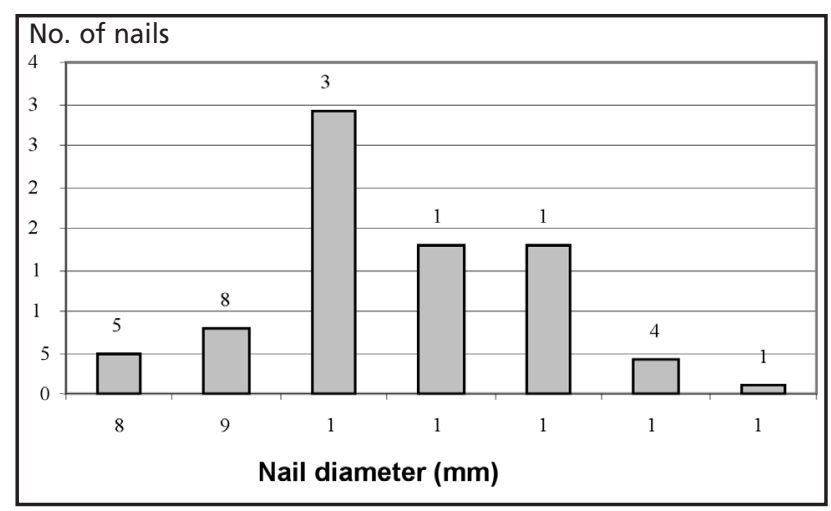

Fig. 3: Diameter of the nails used.

\section{DISCUSSION}

Treatment for femoral shaft fractures varies from plating to unlocked intramedullary nailing and interlocked intramedullary nailing. In 1984, Johnson et $\mathrm{al}^{3}$ published a report comparing treatment of comminuted femoral shaft fracture using roller traction, open unlocked intramedullary nailing with cerclage wire and interlocked intramedullary nailing. He reported that open unlocked nailing with cerclage wire had a high rate of infection, nonunion and shortening (Table I). Similarly, plating of comminuted femoral shaft fracture has proven to have a significantly higher rate of implant failure and nonunion ${ }^{4,5,6,7}$ (Table 4).

Interlocked intramedullary nailing is currently considered the gold standard of treatment for femoral shaft fractures. With its load sharing ability that allows healing by secondary union, refracture has become less common. Unlocked intramedullary nails are still being used for fixation of selected femoral shaft fractures. This method of fixation is not effective in preventing shortening and rotation over the fracture site especially in Winquist type III and IV fractures. Sharma et $a l^{10}$ treated patients with comminuted femoral shaft fractures with closed unlocked intramedullary nails and functional cast bracing for one to four weeks, and reported that only one patient $(1.2 \%)$ had malrotation of more than 10

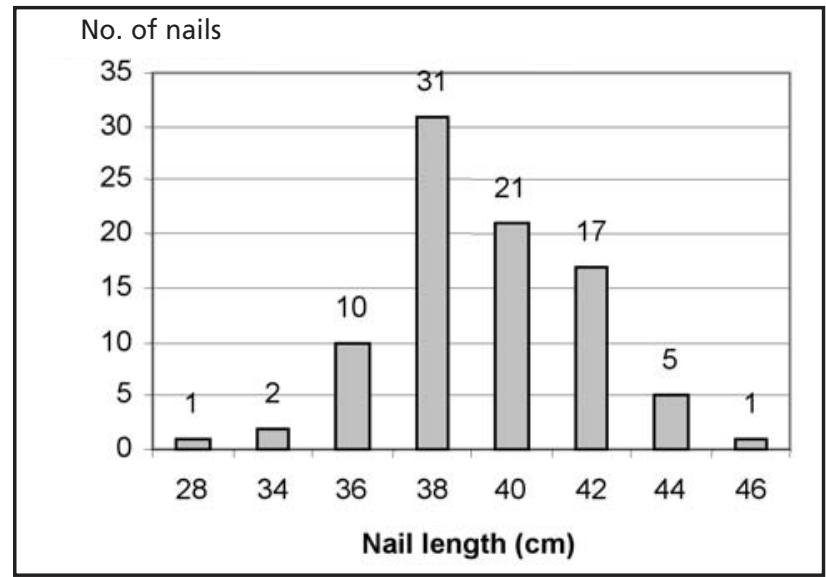

Fig. 2: Length of nails used.

degrees and two $(2.4 \%)$ had shortening more than $2 \mathrm{~cm}$. (Table IV). However, the longer hospitalization and requirement of cast bracing are not appealing to many patients.

In the current study, we found that the conventional unlocked intramedullary nail (Küntscher nail) is still a reliable alternative for Winquist Type I and Type II fractures over the femoral isthmus in that there was a rate of $97.7 \%$ of satisfactory fracture union with an average of 16 weeks to union. The few complications were 2 cases (2.3\%) infection and 2 cases $(2.3 \%)$ of nonunion. This result is comparable to the standard locked intramedullary nail. Unless technical problems such as further comminuting of the fracture during hand reaming or fracture reduction are encountered intraoperatively, stability of the fixation is not a major concern because the medullary canal is hand reamed to the exact size of the implant diameter. The slotted Küntscher nail is hammered into the tight fit canal and converts the compression force into hook stress help to limit rotation instability. Therefore, postoperative rehabilitation is similar to the protocol for locked intramedullary nailing with some modification for pain because of the open surgical wound.

Whether nailing should be closed or open is still debatable. Closed intramedullary nailing is theoretically superior to open nailing because the soft tissue and local circulation at fracture site is not disturbed. Thus, infection and bone union rates are lower in the closed nailing method. In 1969, Rokkanen et $a l^{11}$ compared closed with open femoral nailing and found the closed group had slightly shorter recovery time in term of being able to walk without a cane and also were able to return to work earlier. However, the infection and union rates were same for both groups. Harper ${ }^{12}$ also compared femoral shaft fracture treated with closed and open nailing in 1985 and again found no difference of union and infection rates despite the fact that the open nailing group had larger percentage of open fracture and severe comminuting of the fracture. Their results demonstrated no disadvantages of the open nailing method in terms of 
duration of hospitalization, rate of postoperative complications, time to full weight bearing on the extremity, rate of fracture healing or long term disability.

Open unlocked intramedullary nailing is a good option for selected femoral shaft fractures especially in hospitals where there is no image intensifier or facilities for interlocked intramedullary nailing. Cheaper cost for the open unlocked nailing procedure is another important consideration. In addition, the open procedure eliminates radiation exposure hazards and involves less human resources (i.e., radiographers). Finally the operation room time needed is shorter because less time is required for positioning the patient on the traction table.

\section{CONCLUSION}

Open unlocked intramedullary nailing is a good option for the treatment for Winquist Type I and II femoral fracture around the isthmus. It provides a good union rate and low risk of complications, and is comparable to closed interlocked intramedullary nailing for this group of fractures. 


\section{REFERENCES}

1. Winquist RA, Hansen ST Jr, Clawson DK. Closed intramedullary nailing of femoral fracture: a report of five hundred and twenty cases. J Bone Joint Surg [Am]. 1984; 66-A: 529-39.

2. Hooper GJ, Lyon DW. Closed unlocked nailing for comminuted femoral fractures. J Bone Joint Surg [Br]. 1988; 70-B: 619-21.

3. Johnson KD, Johnston DWC, Parker B. Comminuted femoral shaft fractures; Treatment by roller traction, cerclage wires and an intramedullary nail, or an interlocking intramedullary nail. J Bone Joint Surg [Am]. 1984; 66-A: 1222-35.

4. Reüdi TP, Lüscher N. Results after internal fixation of comminuted fractures of the femoral shaft with DC plates. Clin Orthop. 1979; 138: 74-6.

5. Magerl F, Wyss A, Brunner C, Binder W. Plate osteosynthesis of femoral shaft fractures in adults. Clin Orthop. 1979; 138: 62-73.

6. Cheng JCY, Tse PYT, Chow YYN. The place of the dynamic compression plate in femoral shaft fractures. Injury. 1985; 16: 51934.

7. Thompson F, O'Beirne J, Gallagher J. Fractures of the femoral shaft treated by plating. Injury. 1985; 16: 535-8.

8. Rozbruch SR, Müller U, Gautier E, Ganz R. The evolution of femoral shaft plating technique. Clin Orthop. 1998; 354: 195-208.

9. Wiss DA, Fleming CH, Matta JM, Clark D. Comminuted and rotational unstable fractures of the femur treated with an interlocking nail. Clin Orthop. 1986; 212: 35-47.

10. Sharma JC, Gupta SP, Mathur NC. Comminuted femoral shaft fractures treated by closed intramedullary nailing and functional cast bracing. J Trauma. 1993; 34: 786-91.

11. Rokkanen P, Slätis P, Vankka E. Closed or open intramedullary nailing of femoral shaft fracture? A comparison with conservatively treated cases. J Bone Joint Surg [Br] 1969; 51-B(2): 313-23.

12. Harper MC. Fractures of femur treated by open and closed intramedullary nailing using the fluted rod. J Bone Joint Surg [Am] 1985; 67-A: 699-708. 